Research Articles: Behavioral/Cognitive

\title{
Direct cochlear recordings in humans show a theta rhythmic modulation of auditory nerve activity by selective attention
}

https://doi.org/10.1523/JNEUROSCI.0665-21.2021

Cite as: J. Neurosci 2022; 10.1523/JNEUROSCI.0665-21.2021

Received: 29 March 2021

Revised: 8 November 2021

Accepted: 10 November 2021

This Early Release article has been peer-reviewed and accepted, but has not been through the composition and copyediting processes. The final version may differ slightly in style or formatting and will contain links to any extended data.

Alerts: Sign up at www.jneurosci.org/alerts to receive customized email alerts when the fully formatted version of this article is published.

Copyright @ 2022 Gehmacher et al.

This is an open-access article distributed under the terms of the Creative Commons Attribution 4.0 International license, which permits unrestricted use, distribution and reproduction in any medium provided that the original work is properly attributed. 


\section{Direct cochlear recordings in humans show a theta 2 rhythmic modulation of auditory nerve activity by 3 selective attention}

4 Attentional modulations of the human auditory nerve

5 Quirin Gehmacher ${ }^{1,2 *} \uparrow$, Patrick Reisinger ${ }^{1,2} \uparrow$, Thomas Hartmann ${ }^{1,2}$, Thomas Keintzel ${ }^{3}$,

6 Sebastian Rösch ${ }^{4}$, Konrad Schwarz ${ }^{5}$, Nathan Weisz ${ }^{1,2,6}$

$7 \quad{ }^{1}$ Centre for Cognitive Neuroscience, University of Salzburg, 5020 Salzburg, Austria

$8{ }^{2}$ Department of Psychology, University of Salzburg, 5020 Salzburg, Austria

$9{ }^{3}$ Department of Otorhinolaryngology, Klinikum Wels-Grieskirchen GmbH, 4600 Wels, Austria

$10{ }^{4}$ Department of Otorhinolaryngology, Head and Neck Surgery, Paracelsus Medical University

11 Salzburg, 5020 Salzburg, Austria

$12 \quad{ }^{5}$ MED-EL GmbH, 6020 Innsbruck, Austria

$13{ }^{6}$ Neuroscience Institute, Christian Doppler University Hospital, Paracelsus Medical University

14 Salzburg, 5020 Salzburg, Austria

15 †These authors contributed equally to this work.

*Corresponding author: quirin.gehmacher@sbg.ac.at

17 Number of pages: 29

18 Number of figures: 2

19 Number of tables: 0

20 Number of words - Abstract: 238

21 Number of words - Introduction: 671

22 Number of words - Discussion: 1567

\section{Conflict of interest statement}

K.S. is an employee of MED-EL GmbH. All other authors declare no competing interests.

\section{Acknowledgments}

Q.G. and P.R. are supported by the Austrian Research Promotion Agency (FFG; BRIDGE 1 project "SmartCls"; 871232) and the Austrian Science Fund (FWF; Doctoral College "Imaging their help in recruiting participants. 


\section{Abstract}

The architecture of the efferent auditory system enables prioritization of strongly overlapping spatiotemporal cochlear activation patterns elicited by relevant and irrelevant inputs. So far, attempts at finding such attentional modulations of cochlear activity delivered indirect insights in humans or required direct recordings in animals. The extent to which spiral ganglion cells forming the human auditory nerve are sensitive to selective attention remains largely unknown. We investigated this question by testing the effects of attending to either the auditory or visual modality in human cochlear implant $(\mathrm{Cl})$ users ( 3 female, 13 male). Auditory nerve activity was directly recorded with standard Cls during a silent (anticipatory) cue-target interval. When attending the upcoming auditory input, ongoing auditory nerve activity within the theta range (5$8 \mathrm{~Hz}$ ) was enhanced. Crucially, using the broadband signal $(4-25 \mathrm{~Hz})$, a classifier was even able to decode the attended modality from single-trial data. Follow-up analysis showed that the effect was not driven by a narrow frequency in particular. Using direct cochlear recordings from deaf individuals, our findings suggest that cochlear spiral ganglion cells are sensitive to top-down attentional modulations. Given the putatively broad hair-cell degeneration of these individuals, the effects are likely mediated by alternative efferent pathways as compared to previous studies using otoacoustic emissions. Successful classification of single-trial data could additionally have a significant impact on future closed-loop $\mathrm{Cl}$ developments that incorporate real-time optimization of $\mathrm{Cl}$ parameters based on the current mental state of the user.

Keywords: auditory, auditory nerve, cochlear implants, lateral olivocochlear complex, LOC, 50 selective attention, top-down 


\section{Significance Statement}

52 The efferent auditory system in principle allows top-down modulation of auditory nerve activity, 53 however evidence for this is lacking in humans. Using cochlear recordings in participants 54 performing an audiovisual attention task, we show that ongoing auditory nerve activity in the 55 silent cue-target period is directly modulated by selective attention. Specifically, ongoing 56 auditory nerve activity is enhanced within the theta range when attending upcoming auditory 57 input. Furthermore, over a broader frequency range, the attended modality can be decoded 58 from single-trial data. Demonstrating this direct top-down influence on auditory nerve activity 59 substantially extends previous works that focus on outer hair cell activity. Generally, our work 60 could promote the use of standard cochlear implant electrodes to study cognitive neuroscientific 61 questions. 


\section{Introduction}

Attention describes a process by which sensory information can be prioritized. For all sensory modalities common spatiotemporal cortical activity patterns have been reported, suggesting modality-independent mechanisms to select or ignore features by alterations of oscillatory activity in the alpha (Frey et al., 2014; Mazaheri et al., 2014; Weise et al., 2016) and beta band (Buschman and Miller, 2007; Iversen et al., 2009; Lee et al., 2013). As the auditory system comprises a unique complex subcortical network (Terreros and Delano, 2015; Elgueda and Delano, 2020), cochlear activity can in principle be altered by top-down signals from the auditory cortex via only one extra relay through the superior olivary complex (SOC). However, studying peripheral attentional mechanisms requires special recording and analysis techniques (Elgueda and Delano, 2020) and has therefore been rarely investigated.

Noninvasively, evidence in humans comes from studies on otoacoustic emissions (OAEs), sounds that are generated by outer hair cell $(\mathrm{OHC})$ activity in the cochlea. OHCs are modulated by a pathway from the medial olivocochlear (MOC) system that itself originates in the superior olivary complex (SOC). Spiral ganglion cells making up the auditory-nerve fibers are mainly innervated by connections of the lateral olivocochlear complex (LOC) respectively (Warr and Guinan, 1979; Elgueda and Delano, 2020). Attentional modulations of OAEs can thus be seen as a proxy for subcortical attentional modulations via MOC synapses and have been connected with low-frequency $(<10 \mathrm{~Hz})$ oscillatory mechanisms at the cochlear level during alternating selective attention (Dragicevic et al., 2019), with increases in the theta band ( 6 Hz) when attending to upcoming auditory input during a silent cue-target interval (Köhler et al., 2021).

Further evidence for attention modulations of cochlear activity stems from direct recordings in animals with chronically implanted round-window electrodes, showing decreased auditory nerve action potentials to task-irrelevant click sounds during alternate states of visual attention in cats 
(Oatman, 1971) and during selective attention in chinchillas (Delano et al., 2007). However, whether human auditory nerve activity can be directly modulated via selective attention remains unknown.

While direct recordings are normally not feasible in humans, cochlear implants $(\mathrm{Cl})$ provide a unique opportunity for recording auditory nerve activity. Besides stimulating nerve fibers inside the cochlea, conventional $\mathrm{Cl}$ electrodes are used to measure short responses $(\sim 0.2-0.8 \mathrm{~ms})$ to biphasic pulses, so called electrically evoked compound action potentials (ECAPs), to assure auditory nerve and device functioning during and after surgery (Miller et al., 2008; Ramekers et al., 2014). However, the aforementioned effects of selective attention were reflected in slow oscillatory activity $<30 \mathrm{~Hz}$, which cannot be measured with standard short-latency ECAPs. Assuming as a working hypothesis that selective attention modulates human auditory nerve activity in a similar frequency range, our approach appended short recording windows in a silent cue-target period. This technique allows for discrete sampling of that period within a single trial that can later be processed like standard electroencephalographic (EEG) recordings. Interestingly, $\mathrm{Cl}$ recipients lack the efferent $\mathrm{MOC}$ reflex that leads to cochlear dynamic compression in normal hearing (Wilson et al., 2003; Lopez-Poveda et al., 2016; Marrufo-Pérez et al., 2019). As they additionally show substantial degeneration of OHCs which are further damaged during surgical implant insertion, proposed $\mathrm{Cl}$ recordings during selective attention should mostly reflect modulations of spiral ganglion cell activity via LOC connections (LopezPoveda, 2018) rather than modulations of scattered OHC populations by residual MOC efferents. This argument is strengthened by Liberman and Liberman (2019), who quantified cochlear innervation density by MOC and LOC efferents, highlighting a general paucity of MOC projections that dramatically increases with age whilst LOC populations remain unaffected by this aging-effect. 
Using an audiovisual crossmodal attention task adapted from Hartmann and Weisz (2019, see

111 Figure 1A), we show that ongoing auditory nerve activity in a silent cue-target interval is

A)

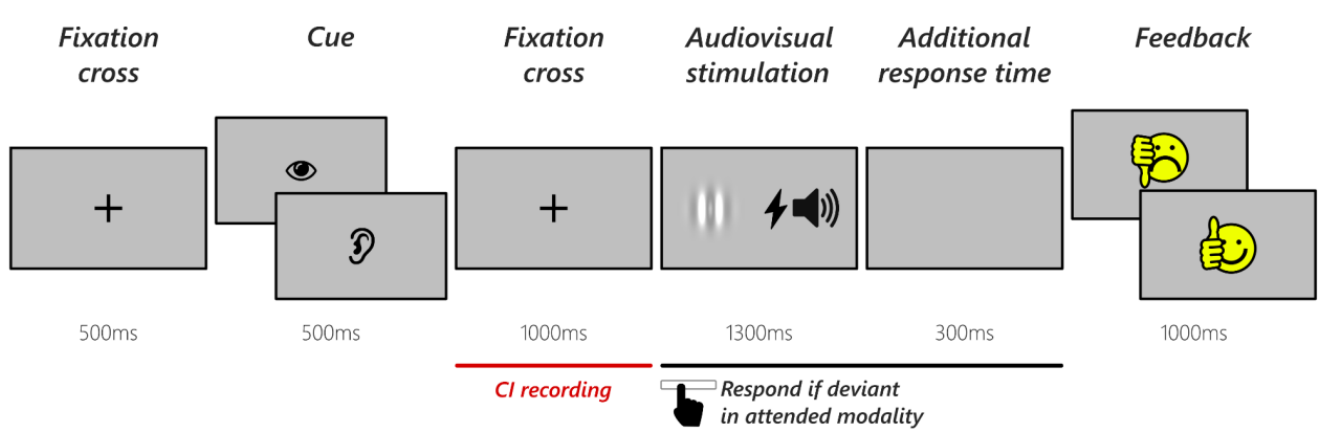

B)

Setup

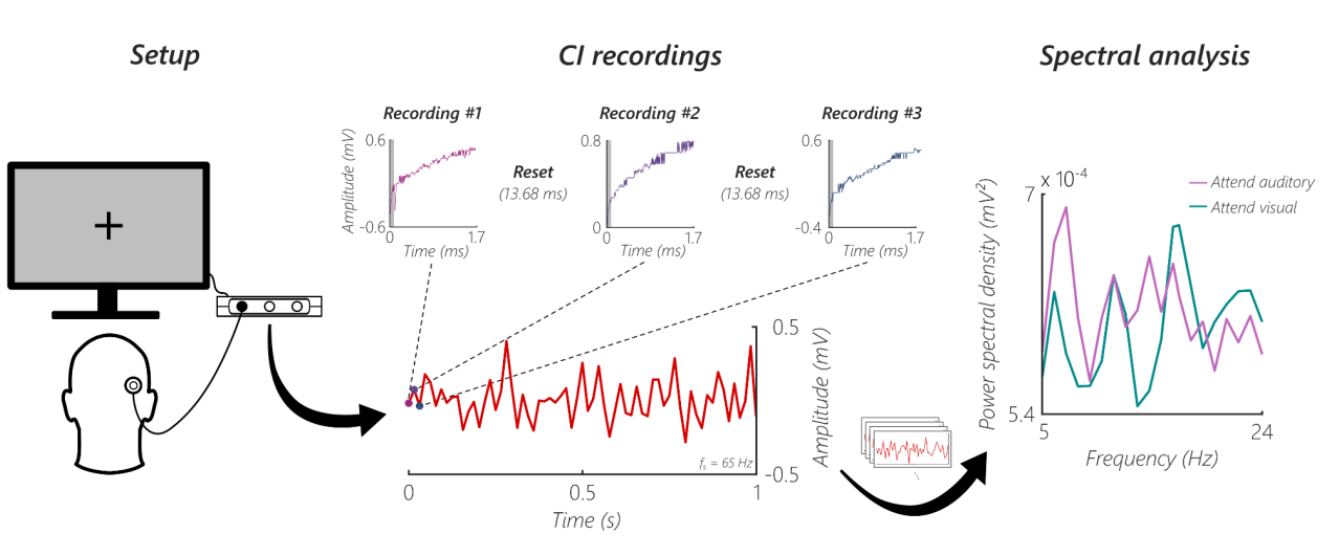

116 modulated by focused attention using standard MED-EL Cls as recording devices (see Figure 1B). In addition to this average condition-level effect, we show that a classifier is even able to decode attended modality on a single-trial basis, which could have important implications for the use of conventional $\mathrm{Cls}$ in a closed-loop system.

Figure 1. Schematic illustration of the crossmodal attention task and $\mathrm{Cl}$ recordings. (A) Each trial started with a fixation cross, followed by a cue indicating either to attend the visual or auditory domain. A second fixation cross appeared and an auditory and visual stimulus were presented afterwards. When the stimulus in the attended modality was deviant (visual: gabor patch tilt, auditory: oddball sound), participants had to respond by pressing the spacebar. The additional response time accounted for trials 
where the gabor patch tilted towards the end of the stimulation. At the end of each trial, feedback was given in the form of a smiley face. The red line denotes the time window where auditory nerve activity was recorded via the $\mathrm{Cl}$. (B) Left: Participants were seated in front of a computer screen and were asked to remove their $\mathrm{Cl}$ processor and coil to replace it with a coil connected to one of the ports of the MAX Programming Interface. Middle: Each recording window was $1.7 \mathrm{~ms}$ long, followed by a $13.68 \mathrm{~ms}$ reset period (three recordings of an exemplary participant are shown). Due to filter artifacts, the first 100 samples from every recording window were discarded (shaded grey area in the recordings). Each recording was averaged and treated as one sample point. By concatenating these single samples, a recording length of 1 second with a sampling frequency $\left(f_{s}\right)$ of $65 \mathrm{~Hz}$ was reached in every trial. Right: Single trials were averaged and spectral analysis was performed separately for the two conditions.

\section{Materials and Methods}

\section{Participants}

21 right-handed $\mathrm{Cl}$ users (4 females, $M_{\text {age }}=57.5, S D_{\text {age }}=11.9$ ) participated in the study, all with a minimum $\mathrm{Cl}$ experience of six months. Participants were recruited via the ear-nose-throat (ENT) departments of the hospitals in Salzburg $(n=10)$ and Wels-Grieskirchen $(n=11)$. Three participants were excluded because of a too weak contact between transmitting $\mathrm{Cl}$ coil and receiver that was required for the study. One participant showed no N1 in recorded ECAPS, which could indicate a measurement problem and was therefore excluded. One participant quit during the session due to concentration problems. This led to a final sample size of 16 participants (3 females, $M_{\text {age }}=53.8, S D_{\text {age }}=12.0$ ). All participants reported no previous neurological or psychiatric disorders, and reported normal or corrected-to-normal vision. All participants signed an informed consent and were reimbursed with 10 Euro per hour. The experimental protocol was approved by the ethics committee of the University of Salzburg and was carried out in accordance with the Declaration of Helsinki. 


\section{Stimuli and experimental design}

The experimental procedure was implemented in MATLAB 8.6 (The MathWorks Inc., Natick, Massachusetts, USA) using custom scripts. Presentation of visual stimuli and response collection was achieved with a previous version (th_ptb; https://gitlab.com/thht/th_ptb) of the Objective Psychophysics Toolbox (o_ptb; Hartmann and Weisz, 2020), which adds an additional class-based abstraction layer in addition to the Psychophysics Toolbox (Version 3.0.14; Brainard, 1997; Pelli, 1997; Kleiner et al., 2007). Cochlear stimulation as well as recording was performed via the MAX Programming Interface, a device which is part of the clinical standard setup that enables control of the implant, together with the Research Interface Box 2 Dynamiclink library (RIB2 DLL provided by the University of Innsbruck, Innsbruck, AT; Litovsky et al., 2017). To ensure accurate stimulus presentation and triggering, timings were measured with the Black Box ToolKit v2 (The Black Box ToolKit Ltd., Sheffield, UK).

Participants were seated in front of a computer screen and were asked to remove their $\mathrm{Cl}$ processor and coil to replace it with a coil connected to the MAX Programming Interface. For bilateral $\mathrm{Cl}$ users, the side with the better subjective hearing performance and/or longer implantation date was used. Primarily the $\mathrm{Cl}$ coil model MAX Coil was used, but if the magnet was too weak to ensure a stable connection, the $\mathrm{Cl}$ coil model MAX Coil S was used. As a first step, the individual electrical hearing threshold was determined with a standard tone with a stimulation frequency of $100 \mathrm{~Hz}$ and a duration of $300 \mathrm{~ms}$. To ensure that the auditory stimulation was at a comfortable level during the experiment, the individual maximum loudness was determined, for the standard and an oddball tone respectively. An oddball tone with the maximum possible stimulation frequency of $9990 \mathrm{~Hz}$ (based on the used phase duration of 30 $\mu$ s per phase for sequential biphasic pulses) and a duration of $300 \mathrm{~ms}$ was used. The described routines were implemented using custom scripts and the Palamedes Toolbox (Prins and Kingdom, 2018). 
Afterwards, as a functionality check of the measurement setup, ECAPs were recorded (Bahmer et al., 2010). ECAPs were biphasic pulses (anodal polarity of the first pulse phase) with a $40 \mu \mathrm{s}$ phase duration and a $147 \mu$ s interpulse interval. In each participant, the first (i.e. most apical) electrode was used for stimulation and the second for recording. Phase amplitudes and amount of ECAPs measured in each participant were defined between the minimum amplitude given by the electrical hearing threshold and the maximum amplitude given by the maximum loudness of the standard tone (phase amplitude: in steps of 9.45 current units (CU); amount: in steps of one).

For the crossmodal attentional task described later, it was necessary that two stimulation frequencies could be distinguished. Because of interindividual differences when hearing with a $\mathrm{Cl}$, it was necessary to adjust these stimulation frequencies for every participant. Participants were asked, after hearing a standard and oddball tone, if the first or the second tone had a higher stimulation frequency. The standard tone had a stimulation frequency of $100 \mathrm{~Hz}$ and a duration of $300 \mathrm{~ms}$. The initial stimulation frequency of the oddball tone (also with a duration of $300 \mathrm{~ms}$ ) was determined by the results of the aforementioned maximum loudness procedure. This procedure was carried out using a Bayesian active sampling protocol to estimate the model parameters of the psychometric function (Kontsevich and Tyler, 1999; Sanchez et al., 2016) and was implemented with the VBA Toolbox (Daunizeau et al., 2014). To define the individual oddball stimulation frequency for the subsequent crossmodal attention task, the algorithm searched for the optimal difference in logarithmic steps from 1 to $9890 \mathrm{~Hz}$ and this value was subsequently added to the standard stimulation frequency. Six participants heard no clear difference and it was necessary to adjust the oddball stimulation frequency manually, with values between 114 and $600 \mathrm{~Hz}$.

The actual experiment was carried out as a crossmodal attention task (see Figure 1A; similar to Hartmann and Weisz, 2019) in six blocks, with 85 trials per block. Each trial started with a 500 
ms fixation cross, followed by a cue that indicated either to attend the auditory or the visual modality. Every block had 43 auditory and 42 visual cues. The cue was a picture of an eye or ear, presented for $500 \mathrm{~ms}$. A second fixation cross appeared for $1000 \mathrm{~ms}$ and the audiovisual stimulation started afterwards. The auditory stimulation consisted of a $300 \mathrm{~ms}$ tone with a stimulation frequency of $100 \mathrm{~Hz}$ and was directly presented via the $\mathrm{Cl}$ coil. The visual stimulation was a vertically oriented gabor patch (spatial frequency: 0.01 cycles/pixel, sigma: 60 pixels, phase: $90^{\circ}$ ), presented for $1300 \mathrm{~ms}$ in the center of the screen. In every block, 8 trials were randomly chosen as visual oddball trials. Independently, another 8 trials were chosen to be auditory oddball trials. Therefore, it was possible that a trial was a visual and auditory oddball trial simultaneously. In visual oddball trials, the gabor patch tilted $10^{\circ}$ to the left, with a random onset. In auditory oddball trials, a $300 \mathrm{~ms}$ tone with the individual oddball stimulation frequency was presented. Participants had to press the spacebar if the current trial had an oddball in the cued domain. To account for trials where the visual oddball onset was towards the end of the stimulation, an additional response time of $300 \mathrm{~ms}$ was provided. After each trial, feedback in the form of a smiley face displayed for $1000 \mathrm{~ms}$, indicated if the response was correct or not. To ensure that participants understood the task and responded appropriately, they completed one block as a practice run before the actual experiment. The total duration of the experiment was about 90 minutes including breaks and preparation.

\section{Recording of auditory nerve activity}

We exploit the ability of Cls to record electrical activity from the cochlea in short time windows, but in contrast to previous approaches (Mc Laughlin et al., 2012; Abbas et al., 2017), in a silent cue-target period. Using a custom developed MATLAB toolbox to abstract MAX Programming Interface commands, we recorded auditory nerve activity via the $\mathrm{Cl}$ electrode. In every participant, the first (i.e. most apical) electrode was used for the recordings. Each recording window was $1.7 \mathrm{~ms}$ long, followed by a $13.68 \mathrm{~ms}$ reset period resulting in a sampling frequency 
of $65 \mathrm{~Hz}$ (see Figure 1B; $1.7 \mathrm{~ms}$ recording $+13.68 \mathrm{~ms}$ reset time). The sampling rate of each

$2221.7 \mathrm{~ms}$ recording window was 1.2 Mhz (i.e. 2048 sample points in each recording window). The 223 technical specifics of the measurement system added a random offset to each of the recordings 224 (Gaussian noise, $S D=0.4 \mathrm{mV}$ ). Because of the USB connection between the computer and the 225 MAX Programming Interface, the start of the first recording window had a jitter of $27 \mathrm{~ms}$, but the system sent a highly precise trigger when it started. Due to technical limitations, it was not possible to record and stimulate simultaneously. We performed recordings in the $1000 \mathrm{~ms}$ prestimulus window (see red line in Figure 1A).

Data preprocessing

The raw data was analyzed in MATLAB 9.8 (The MathWorks, Natick, Massachusetts, USA).

Due to filter artifacts (using the standard filter from the used RIB2 package), the first 100 samples $(=0.083 \mathrm{~ms})$ from every recording window were discarded. Afterwards, the recording was averaged and treated as one sample point. By repeating these steps for every window and concatenating the single samples, a recording length of 1 second with a sampling frequency of $65 \mathrm{~Hz}$ was reached. The data was further preprocessed with the FieldTrip toolbox (revision ea6897bb7; Oostenveld et al., 2010) and a bandpass filter between 4 and $25 \mathrm{~Hz}$ was applied (hamming-windowed sinc FIR filter, onepass-zerophase, order: 424, transition width: $0.5 \mathrm{~Hz}$ ). For one participant, 15 trials had to be rejected because the $\mathrm{Cl}$ coil fell off during the last trials of one block. Only trials with a correct response were analyzed, which were on average 488 trials $(S D=15.8)$. The number of correct trials was not significantly different between the two conditions (see Behavioral results).

Next, data was demeaned, detrended and power spectral density (PSD) from 4 to $25 \mathrm{~Hz}$ was 244 computed on the whole 1000 ms prestimulus window ('mtmfft' implementation in FieldTrip with a 
245

246

247

248

249

250

251

252

253

254

255

256

257

258

259

260

261

262

263

264

265

266

267

268

Hann window) separately for the two conditions. For Figure 2A, no bandpass filter was applied, condition-specific power spectra were smoothed (five-point moving average), grand-averaged, and corrected error bars for within-subjects designs were calculated (O'Brien and Cousineau, 2014).

We defined two frequency bands of interest (FOI), theta $(5-8 \mathrm{~Hz})$ and alpha $(9-13 \mathrm{~Hz})$. Theta was selected because of previous work on OAEs that showed attentional modulations in this FOI (Dragicevic et al., 2019; Köhler et al., 2021). On a cortical level, previous work showed that auditory alpha activity reflects attentional processes (Weisz et al., 2011, 2014; Müller and Weisz, 2012; Frey et al., 2014; Mazaheri et al., 2014; Weise et al., 2016). Therefore, we decided to analyze this FOI at the cochlear level.

\section{Decoding analysis}

For decoding of attended modality on a single-trial basis, we performed k-nearest neighbors (kNN) classification of single-trial power spectra using scikit-learn (Version 0.23.1 running on Python 3.7.7; Pedregosa et al., 2011) separately for a broadband signal (4-25 Hz) followed by standard frequency bands associated with selective attention (theta: 5-8 Hz, alpha: 9-13 Hz, beta: 14-24 Hz). We decided to use the kNN classification approach as data was recorded from a single $\mathrm{Cl}$ channel over a one second period resulting in low numbers of features (i.e. frequency points per band), a classification problem usually solved better by a kNN approach (Eisa et al., 2018).

At first, a subject's data was standardized to unit variance and zero mean. For the classification process of each subject, the best number of neighbors was determined by searching the hyperparameter space for the best cross-validation (CV) score of a kNN model using the implemented GridSearchCV function with a 2-fold CV on shuffled class samples (StratifiedKFold(shuffle=True)) that was fit to the data for every FOI. Our decision for a 2-fold CV 
269

270

271

272

273

274

275

276

277

278

279

280

281

282

283

284

285

286

287

288

289

290

291

292

was based on recommendations in case of low sample/effect size data (Jamalabadi et al., 2016). The numbers of neighbors to use during the gridsearch were defined as ranging from one to $10 \%$ of trials in the dataset in odd numbers $(1$, trials $/ 10$, stepsize $=2)$ to avoid the conflict of even neighbors in a two-class problem (attend auditory vs. visual).

\section{Statistical analysis}

\section{Cluster-based permutation statistics of the power spectra}

To test the hypothesis that power was higher when the auditory domain was attended, statistical testing of PSD was performed with a cluster-based permutation test (dependent samples t-test, 10000 randomizations, one-tailed; Maris and Oostenveld, 2007). We averaged the theta and alpha FOI and tested them separately.

\section{Decoding analysis statistics}

Given the novel approach, we could not exclude that the classifier would pick up on a few outlying data points. In order to address this issue explicitly, the classifier was tested on the same noisy data, albeit with randomly shuffled condition labels. Samples were thus classified and tested for significance with the best scoring number of neighbors in a 1000 random permutation test and the aforementioned 2-fold CV procedure. The resulting Observed and Chance accuracy values (where chance level was calculated as the mean accuracy of the 1000 random permutation scores) for every FOI were then statistically tested using pingouin (Version 0.3.8 running on Python 3.7.7; Vallat, 2018). In a first step, to test whether auditory nerve modulation was generally reflected within classification results, broadband values (Observed vs. Chance) were compared using a one-sided t-test. Then, classification results of all four FOI were compared in a two-factor repeated measures ANOVA with the factors FOI (broadband, theta, alpha, beta) and Type (Observed vs. Chance) to check whether the attention effect was driven by one of the predefined FOI. Finally, theta, alpha, and beta bands were also tested for 
293

294

295

296

297

298

299

300

301

302

303

304

305

306

307

308

309

310

311

312

313

314

significant differences during focused attention computing three one-sided t-tests with respective values (Observed vs. Chance).

Code Accessibility

The data and code necessary for statistical analysis and generating the figures are available at the corresponding author's GitLab repository (https://gitlab.com/qubitron).

\section{Results}

Sixteen $\mathrm{Cl}$ users performed a crossmodal attention task (similar to Hartmann and Weisz, 2019) where attention had to be focused on an upcoming auditory or visual stimulus (see Figure 1A). Auditory nerve activity was recorded directly via their first (i.e. most apical) $\mathrm{Cl}$ electrode in the silent cue-target interval. We calculated the power spectral density of the signal and compared the two conditions (attend auditory vs. visual) in the theta and alpha band. Afterwards, a classifier was utilized to decode the attended modality on a single-trial basis using the broadband signal and frequency bands typically associated with selective attention (theta, alpha, beta).

\section{Behavioral results}

Participants gave a correct response in $96 \%(S D=2.7 \%)$ of all trials. The number of correct trials did not differ significantly between the two conditions, according to a dependent sample ttest (auditory: $M=245(S D=9.8)$; visual: $M=242(S D=8.8) ; t(15)=1.32, p=0.21, d=0.33$ ). When there was an oddball in the cued domain, a correct response was given in $75 \%$ ( $S D=$ $19.0 \%$ ) of the trials. In the auditory condition, the percentage of correct oddball trials was $72 \%$ $(S D=30.8 \%)$ and in the visual condition $78 \%(S D=14.2 \%)$. Overall, the behavioral findings suggested that participants performed the task in a compliant manner. 
316 In a first analysis step, we calculated the broadband PSD from 4-25 Hz, separately for each 317 condition (attend auditory/attend visual). The resulting power spectra (see Figure 2A) by 318 themselves showed no clear peaks, however the grand average condition contrast spectrum 319 indicates differences that are mainly centered in distinct frequency ranges. Based on previous 320 OAE and M/EEG work (Mazaheri et al., 2014; Köhler et al., 2021), we statistically compared the 321 two conditions in the theta (averaged between $5-8 \mathrm{~Hz}$ ) and alpha frequency band (averaged between 9-13 Hz; Figure 2B). A cluster-based permutation test in the theta frequency band showed that prestimulus power is higher when attending the auditory domain $\left(p=10.00 \mathrm{e}^{-05}, d=\right.$ 0.49). No cluster was found in the alpha frequency band $(p=1.00, d=0.20)$. Given the distribution of the individual average power in both $\mathrm{FOI}$, a rather high interindividual variability can be seen. In addition, we also tested the beta frequency band (averaged between $14-24 \mathrm{~Hz}$ ), where no cluster was found ( $p=1.00, d=-0.16)$.

We further wanted to investigate whether the effect in the theta band was related to behavioral performance. Correlating individual average theta power in each condition with behavioral performance showed a very small and non-significant relationship (auditory: $r=0.019, p=$ 0.944; visual: $r=0.096, p=0.723$ ). A potential explanation could be the rare occurrence of oddball trials (see Materials and Methods), resulting in a very high overall behavioral performance (auditory: $M=96 \%, S D=3.1 \%$; visual: $M=95 \%, S D=3.6 \%$ ).

The results so far showed that selective attention modulates directly recorded cochlear activity, with the effect being in particular pronounced in the theta frequency range: attending to an upcoming auditory stimulus resulted in higher power recorded from the $\mathrm{Cl}$ electrode. 
A) Prestimulus power

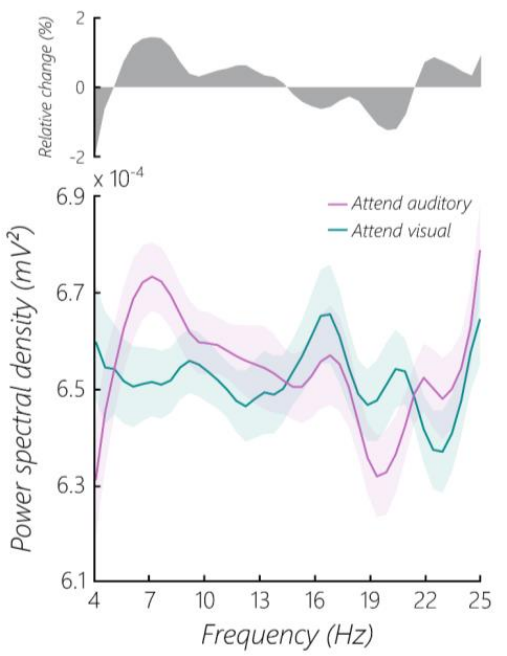

B)

Auditory vs. Visual

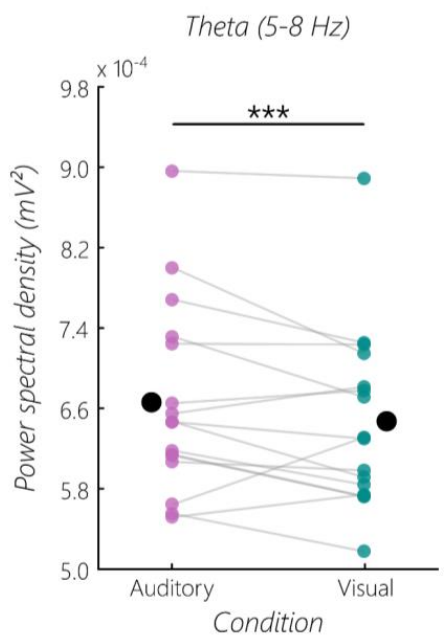

Alpha $(9-13 \mathrm{~Hz})$

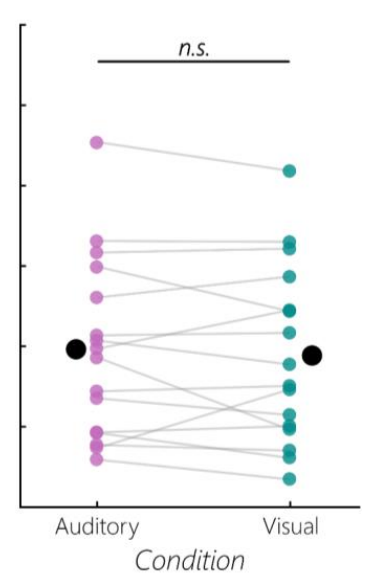

C)

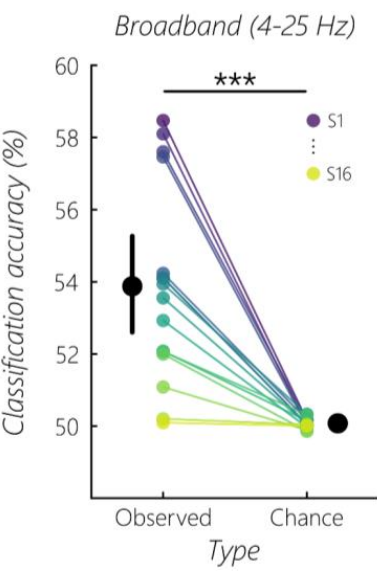

$k N N$-Classification performance

Theta $(5-8 \mathrm{~Hz})$

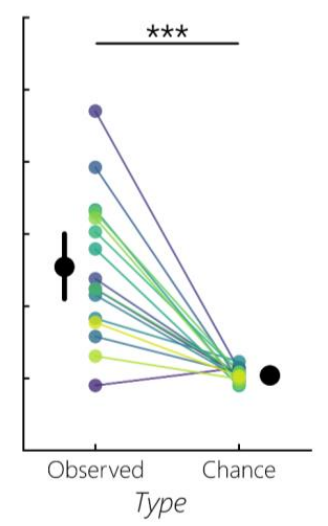

Alpha $(9-13 \mathrm{~Hz})$

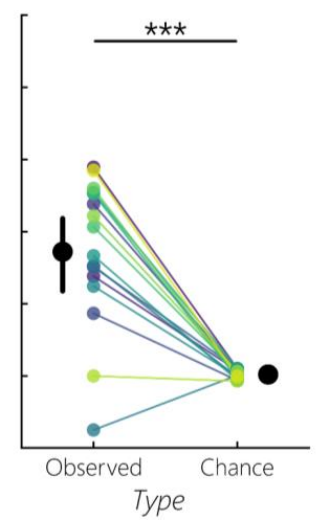

$\operatorname{Beta}(14-24 \mathrm{~Hz})$

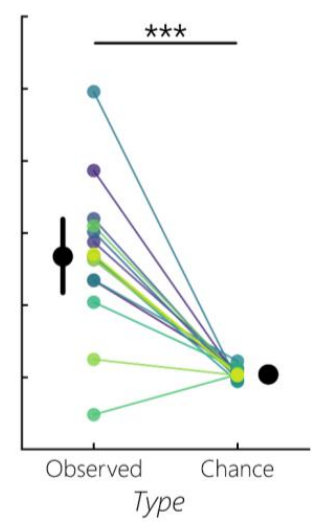

Figure 2. Prestimulus power modulations and decoding of selective attention. (A) Grand average prestimulus power spectra from $4-25 \mathrm{~Hz}$ when attending the auditory or visual domain. The top panel indicates the relative change between the auditory and visual domain. The shaded areas in the bottom panel represent the standard error of the mean for within-subjects designs (O'Brien and Cousineau, 2014). (B) Average prestimulus power in the theta and alpha band, separated by the two conditions. 
344

averaged theta FOI resulted in a statistically significant difference when testing the hypothesis that performance is higher when attending the auditory domain $\left(p=10.00 \mathrm{e}^{-05}, d=0.49\right)$. No cluster was found in the alpha band $(p=1.00, d=0.20)$. The asterisks indicate a statistically significant difference $(\mathrm{n} . \mathrm{s}$. $=$ not significant). (C) A kNN-Classifier was used to decode attended modality from single-trial prestimulus power spectra. Resulting Observed accuracies were contrasted with respective Chance levels of a random permutation test for all FOI. Contrasts revealed significant $(p<0.001)$ decoding performance throughout spectra with fairly similar effects (broadband: $t(15)=5.60, p=2.60 \mathrm{e}^{-05}, d=1.96$; theta: $t(15)=$ 5.83, $p=1.70 \mathrm{e}^{-05}, d=2.11$; alpha: $t(15)=6.78, p=3.00 \mathrm{e}^{-06}, d=2.34$; beta: $t(15)=6.40, p=6.00 \mathrm{e}^{-06}, d=$ 2.33) on a group level (represented by black dots; error bars $=95 \% \mathrm{Cl}$ ). However, on a single-subject level, the attention effect was most pronounced for individually specific FOI, resulting in significant above chance decoding for 12 out of 16 subjects. As indicated in the top right of the broadband column (S1: purple, S16: yellow), each subject is represented by the same color in all four FOI columns.

\section{Auditory nerve as origin of the signal}

Our results so far suggest a theta rhythmic modulation of human auditory nerve activity by selective attention, with increased theta activity when attending an upcoming auditory stimulus. However, one concern of the recording approach was the origin of the recorded signal being actually any cortical source instead of auditory nerve activity. Even though electrode configurations with close (extracochlear) reference on the implant housing should already pick up quite local sources, an empirical approach was needed to exclude possible effects of volume conduction. To show that our effects truly resemble auditory nerve modulations, we exploited an additional EEG dataset that was simultaneously recorded to the $\mathrm{Cl}$ signal for one of the participants. Addressing this issue, we cross-correlated the $\mathrm{Cl}$ signal with the EEG signals of 55 electrodes (9 had to be excluded due to their location directly above the subject's implant). If signals at the $\mathrm{Cl}$ were due to volume conduction, this should go along with strong crosscorrelations at zero-lag. To test the absence of this instantaneous volume conduction effect, we 
calculated the average Bayesian Pearson correlation between $\mathrm{Cl}$ and EEG signals for 510 trials using the 'bayesFactor' package for Matlab (see https://klabhub.github.io/bayesFactor). The implemented correlation function uses the Jeffreys-Zellner-Siow (JZS) prior as proposed for Bayesian hypothesis test for correlational analysis by Wetzels \& Wagenmakers (2012). We report In-transformed Bayes factors, $\ln \left(B F_{10}\right)$, where values $>1.1$ would mean substantial evidence for volume conduction and values $>2.3$ would mean strong evidence for volume conduction (Jeffreys, 1998). Results of this analysis show no evidence for volume conduction according to $\ln \left(\mathrm{BF}_{10}\right): M=-0.98(S D=0.40)$, with values ranging from -1.60 to 0.20 between all electrodes. Respective r-values show a mean of $r=3.46 \mathrm{e}^{-04}(S D=0.0074)$, ranging between 0.016 and 0.011 over all channels. The analysis substantially strengthens our argument that the attentional effects are genuinely recorded from the auditory nerve.

\section{Attended modality can be decoded from single-trial $\mathrm{Cl}$ recordings}

We used prestimulus power spectra for a kNN-Classifier to show that attention modulation of ongoing auditory nerve activity in humans is even reflected in single-trial $\mathrm{Cl}$ recordings. To ensure that the classifier was able to differentiate auditory nerve activity when attending the auditory compared to the visual domain in general, we calculated a t-test between Observed classification accuracies and respective Chance levels of broadband power spectra, showing that this attention effect was decodable significantly above chance $\left(t(15)=5.60, p=2.60 \mathrm{e}^{-05}, d\right.$ = 1.96; Figure 2C). Given the significant difference over a broad frequency range, we were further interested in whether this attention effect was driven by one of the FOI usually connected with selective attention in OAE and M/EEG studies. We therefore calculated a two-factor repeated measures ANOVA to compare the effect of selective attention on kNN-Classification accuracy for different FOI (broadband, theta, alpha, beta) and Types (Observed, Chance). Results show no significant effect of $\mathrm{FOI}\left(F(3,45)=0.37, p=0.78, \eta_{\mathrm{p}}^{2}=0.02\right)$, yet show a significant effect for Observed vs. Chance accuracies $\left(F(1,15)=136.55, p=6.21 \mathrm{e}^{-09}, \eta_{\mathrm{p}}{ }^{2}=\right.$ 
394

395

396

397

0.90), with higher accuracies for Observed $(M=0.53)$ than Chance $(M=0.50)$ levels. No FOI $x$ Type interaction on decoding results was found $\left(F(3,45)=0.36, p=0.78, \eta_{p}^{2}=0.02\right)$. As a main effect of Type and no interaction of FOI x Type indicated that selective attention can be decoded from all FOl separately, in addition we computed three t-tests for theta, alpha, and beta bands contrasting respective Observed and Chance levels. For all three FOI a significant difference was found for selective attention decoding (theta: $t(15)=5.83, p=1.70 \mathrm{e}^{-05}, d=2.11$; alpha: $t(15)=6.78, p=3.00 \mathrm{e}^{-06}, d=2.34$; beta: $t(15)=6.40, p=6.00 \mathrm{e}^{-06}, d=2.33$; Figure 2C) Additionally, random permutation tests of kNN classification within all four different FOI gave insights into single-subject decoding performance across the different frequency spectra. Independent of $\mathrm{FOI}$, an overall number of 12 subjects (i.e. $75 \%$ of the sample) showed significant $(p<0.05)$ above chance decoding of focused attention during the silent cue-target interval.

\section{Discussion}

The efferent auditory system comprises a complex arrangement of subcortical pathways, which can alter cochlear activity by top-down signals (Terreros and Delano, 2015; Elgueda and Delano, 2020). Profound evidence supports the notion of altered oscillatory neural activity by selective attention on a cortical level within the alpha (Frey et al., 2014; Mazaheri et al., 2014; Weise et al., 2016) and beta band (Buschman and Miller, 2007; Iversen et al., 2009; Lee et al., 2013). Much less is known for subcortical structures along the efferent pathway, especially when it comes to the human cochlea as special recording and analysis techniques are required (Elgueda and Delano, 2020). So far, investigating attentional modulation of cochlear activity in humans had to rely on indirect recordings of OAEs, a noninvasive approach for measuring $\mathrm{OHC}$ activity. Recent evidence suggests slow modulations $(<10 \mathrm{~Hz})$ of cochlear activity (Dragicevic et al., 2019) that is even enhanced during a silent cue-target period when attending the auditory 
modality (Köhler et al., 2021). However, studying OAEs cannot address direct modulation of auditory nerve activity since spiral ganglion cells are efferently innervated by a separate, lateral pathway. In contrast to the MOC, most LOC fibers project to the ipsilateral cochlea (Robertson, 1985) and are evenly distributed from the apical to the basal end (Guinan, 1996). In humans, little is known about their function in sound processing since its unmyelinated axons are difficult to electrically stimulate and record from (Guinan, 2018), while measured responses are inconclusive about MOC or LOC origin. To our knowledge, attentional modulation via the LOC remains completely unknown, as direct recordings of auditory nerve activity are normally not feasible in humans. Given the absence of the efferent MOC reflex in $\mathrm{Cl}$ recipients (Wilson et al., 2003; Lopez-Poveda et al., 2016; Marrufo-Pérez et al., 2019) in addition to substantial OHC degeneration, as well as the overall scarcity of efferent MOC projections (Liberman and Liberman, 2019), potential alterations of respective auditory nerve activity in a selective attention paradigm should largely reflect top-down signals from the LOC (Lopez-Poveda, 2018). Crucially, LOC innervation density peaks at the apical end of the cochlea, whereas MOC projections peak in mid-cochlear regions (Liberman and Liberman, 2019). As we exclusively recorded from the most apical electrode, the reported effects should most definitely reflect modulations of apical LOC innervations. Our results show that ongoing auditory nerve activity is top-down modulated, hereby putatively suggesting a role of the LOC pathway in selective attention. Future applications that are able to simultaneously record from multiple $\mathrm{Cl}$ electrodes could exploit the different electrode positions along the cochlea and draw conclusions about frequency specific terminal distributions of LOC efferents. Setups with bilateral CI recordings could even address the LOC's role in analysis of interaural differences in frequency and intensity, as assumed by Ciuman (2010).

Importantly, we ruled out any cortical source to drive the demonstrated effects as Somers et al. (2021) recently showed that tailored Cls can be used to intentionally obtain evoked potentials 
from the auditory cortex with a recording setup that is fairly comparable with only small differences to the one used in this study. Whilst Somers et al. used reference electrodes located on the temporal muscle, MED-EL implants have reference and ground electrodes in the actual implant housing. It was therefore important to rule out any cortical origin with additional analysis. Cross-correlation of simultaneously recorded $\mathrm{Cl}$ and $\mathrm{EEG}$ electrodes for one participant clearly showed the absence of any instantaneous correlations which would be caused by volume conduction.

While cortical and OAE-based measures suggest attention-related effects in distinct frequency bands (Mazaheri et al., 2014; Köhler et al., 2021), our results are mixed in this respect. The broadband frequency analysis of the prestimulus interval showed no clear peaks (Figure 2A). This, however, may also be the result of low signal-to-noise ratios (SNRs), as commercial Cls are so far not optimized to do these kinds of continuous electrophysiological recordings. Indeed, a grand average of the condition differences points to maximal effects in a frequency range overlapping with the one reported by Köhler et al. (2021), resulting in enhanced theta power while attending to the auditory modality (Figure 2B). This result corroborates our previous finding using a similar paradigm, where ongoing OAEs in the theta band $(\sim 6 \mathrm{~Hz})$ were enhanced while attending an upcoming auditory stimulus (Köhler et al., 2021). We found no selective attention effect in the alpha nor in the beta band in concordance with aforementioned studies of otoacoustic activity. It is therefore possible that these frequency bands do not play a central role in selective attention at the peripheral level. A caveat of the present study concerns a possible involvement of the delta band that cannot be ruled out by the analyzed frequency range of 4-25 $\mathrm{Hz}$ due to the restricted $1000 \mathrm{~ms}$ recording periods. Further studies with an optimized recording setup will be necessary to address this issue. However, the dominance of theta rhythmic modulations in the auditory periphery across the reported studies are striking. Arguably, feature selection on a cochlear level shares top-down mechanisms with working memory (WM) 
prioritization processes reflected within the theta band (Riddle et al., 2020). In this context, selective attention could support WM processes in its ability to exclude irrelevant information, i.e. WM filtering efficiency (Arnell and Stubitz, 2010) or expectation-driven WM benefits (Bollinger et al., 2010). This could explain our results with regards to predominance of theta oscillations. However, we think that condition-related increases in the theta band in the current study mainly reflect processes of selective attention rather than visual WM since the cues were fairly similar and the task not demanding in terms of WM capacity. This idea should be further explored in future studies that additionally manipulate memory load and assess WM performance outcomes and how this is affected by individual theta in-/decreases already at a cochlear level. This becomes especially relevant as Marcenaro et al. (2021) just recently established a link between visual WM and acoustic suppression of distortion product OAEs, suggesting top-down modulations of cochlear responses via MOC efferents during visual WM periods.

Building upon conventional analyses of condition-level fast Fourier transform (FFT) averages, we decided to use single-trial frequency spectra to classify anticipatory attentional focus during the silent cue-target period. With this approach we aimed to get more detailed insight into finegrained differences between attentional states coded within modulations of direct cochlear recordings that could be missed by condition-level averaging approaches and indirect OAE measurements. Strikingly, classification of the broadband signal (4-25 Hz) revealed significantly improved differentiation of attended modality compared to the average condition-level effect of the FFT results (Figure 2C). Follow-up analysis showed that the performance was not driven by one of three FOI (theta, alpha, beta) usually associated with selective attention, but instead it revealed that the contribution of each of these frequency bands to broadband classification was fairly similar. However, the decoding approach allowed for additional insight into single-subject classification performance and showed high interindividual variability in terms of an optimal 
493

494

495

496

497

498

499

500

501

502

503

504

505

506

507

508

509

510

511

512

513

514

515

516

517

spectral frequency band. It remains to be determined whether this effect is driven by local idiosyncrasies at the peripheral level (e.g. synaptic connections between LOC and spiral ganglion cells) or even involves particular activity patterns at higher hierarchical levels. Independent of the precise origins of our effects observed at the auditory nerve, the decoding results open up avenues to future developments towards closed-loop Cls that incorporate mental states of the recipient into adaptive stimulation in real time. As we show, a classifier could use the frequency information of this signal to anticipate the attentional state of the recipient. Future research will need to address which cognitive states can be decoded directly at the auditory nerve and how this information could be exploited in a closed-loop $\mathrm{Cl}$ setup. In this regard, overall classification results might seem rather small with accuracies ranging between $50 \%-60 \%$, yet they are comparable to many others in the field of cognitive neuroscience (see Varoquaux et al., 2017, for a review on decoding in neuroimaging). Considering that the results are based on simplistic decoding of noisy single-channel data, the reported accuracies are highly valuable and most importantly interpretable. Future approaches with improved recording setups would most probably allow for higher decoding accuracies as they were necessary for functional closed-loop systems. Additionally, albeit substantial interindividual variability, the classifier yielded accuracies at a quite consistent above chance level. It remains an open question to what extent interindividual differences are functionally relevant. In the current study, we did not observe any relation to behavioral performance, however this may be due to the simplistic task leading to a ceiling effect obscuring associations.

In summary, this study shows that individuals with a $\mathrm{Cl}$ form a model population to deepen our understanding of how cognition can leverage the efferent auditory system to modulate auditory input at the earliest stages of processing. To the best of our knowledge, our study is the first to investigate attentional effects on activity recorded directly from the auditory nerve in humans. We confirm and extend previous indirect measurements, suggesting attentional modulations in 
the theta frequency range. Importantly, we also show that selective attention can be decoded above chance at a single-trial and even individual level. Previous reports on attentional modulations of cochlear activity relied on OAEs, which are driven by the MOC pathway. Our results strongly suggest that the LOC pathway can also be exploited in a top-down fashion to affect spiral ganglion cells directly.

\section{References}

Abbas PJ, Tejani VD, Scheperle RA, Brown CJ (2017) Using Neural Response Telemetry to Monitor Physiological Responses to Acoustic Stimulation in Hybrid Cochlear Implant Users. Ear Hear 38:409.

Arnell KM, Stubitz SM (2010) Attentional blink magnitude is predicted by the ability to keep irrelevant material out of working memory. Psychol Res 74:457-467.

Bahmer A, Peter O, Baumann U (2010) Recording and analysis of electrically evoked compound action potentials (ECAPs) with MED-EL cochlear implants and different artifact reduction strategies in Matlab. J Neurosci Methods 191:66-74.

Bollinger J, Rubens MT, Zanto TP, Gazzaley A (2010) Expectation-Driven Changes in Cortical Functional Connectivity Influence Working Memory and Long-Term Memory Performance. J Neurosci 30:14399-14410.

Brainard DH (1997) The Psychophysics Toolbox. Spat Vis 10:433-436.

Buschman TJ, Miller EK (2007) Top-Down Versus Bottom-Up Control of Attention in the Prefrontal and Posterior Parietal Cortices. Science 315:1860-1862.

Ciuman RR (2010) The Efferent System or Olivocochlear Function Bundle - Fine Regulator and Protector of Hearing Perception. Int J Biomed Sci IJBS 6:276-288.

Daunizeau J, Adam V, Rigoux L (2014) VBA: A Probabilistic Treatment of Nonlinear Models for Neurobiological and Behavioural Data. PLOS Comput Biol 10:e1003441. 
Delano PH, Elgueda D, Hamame CM, Robles L (2007) Selective Attention to Visual Stimuli Reduces Cochlear Sensitivity in Chinchillas. J Neurosci 27:4146-4153.

Dragicevic CD, Marcenaro B, Navarrete M, Robles L, Delano PH (2019) Oscillatory infrasonic modulation of the cochlear amplifier by selective attention. PLOS ONE 14:e0208939.

Eisa DA, Taloba Al, Ismail SSI (2018) A Comparative Study on using Principle Component Analysis with different Text Classifiers. Int J Comput Appl 180:1-6.

Elgueda D, Delano PH (2020) Corticofugal modulation of audition. Curr Opin Physiol 18:73-78.

Frey JN, Mainy N, Lachaux J-P, Müller N, Bertrand O, Weisz N (2014) Selective Modulation of Auditory Cortical Alpha Activity in an Audiovisual Spatial Attention Task. J Neurosci 34:6634-6639.

Guinan JJ (1996) Physiology of Olivocochlear Efferents. In: The Cochlea (Dallos P, Popper AN, Fay RR, eds), pp 435-502 Springer Handbook of Auditory Research. New York, NY: Springer.

Guinan JJ (2018) Olivocochlear efferents: Their action, effects, measurement and uses, and the impact of the new conception of cochlear mechanical responses. Hear Res 362:38-47.

Hartmann T, Weisz N (2019) Auditory cortical generators of the Frequency Following Response are modulated by intermodal attention. Neurolmage 203:116185.

Hartmann T, Weisz N (2020) An introduction to the Objective Psychophysics Toolbox. Front Psychol 11.

Iversen J, Repp B, Patel A (2009) Top-Down Control of Rhythm Perception Modulates Early Auditory Responses. Ann N Y Acad Sci 1169:58-73.

Jamalabadi H, Alizadeh S, Schönauer M, Leibold C, Gais S (2016) Classification based hypothesis testing in neuroscience: Below-chance level classification rates and overlooked statistical properties of linear parametric classifiers. Hum Brain Mapp $37: 1842-1855$. 
567

568

569

570

571

572

573

574

575

576

577

578

579

580

581

582

Jeffreys SH (1998) The Theory of Probability, Third Edition. Oxford, New York: Oxford University Press.

Kleiner M, Brainard D, Pelli D (2007) What's new in Psychtoolbox-3? Perception 36:1-16.

Köhler MHA, Demarchi G, Weisz N (2021) Cochlear activity in silent cue-target intervals shows a theta-rhythmic pattern and is correlated to attentional alpha and theta modulations. BMC Biol 19:48.

Kontsevich LL, Tyler CW (1999) Bayesian adaptive estimation of psychometric slope and threshold. Vision Res 39:2729-2737.

Lee JH, Whittington MA, Kopell NJ (2013) Top-Down Beta Rhythms Support Selective Attention via Interlaminar Interaction: A Model. PLOS Comput Biol 9:e1003164.

Liberman LD, Liberman MC (2019) Cochlear Efferent Innervation Is Sparse in Humans and Decreases with Age. J Neurosci 39:9560-9569.

Litovsky RY, Goupell MJ, Kan A, Landsberger DM (2017) Use of Research Interfaces for Psychophysical Studies With Cochlear-Implant Users. Trends Hear $21: 2331216517736464$

Lopez-Poveda EA (2018) Olivocochlear Efferents in Animals and Humans: From Anatomy to Clinical Relevance. Front Neurol 9.

Lopez-Poveda EA, Eustaquio-Martín A, Stohl JS, Wolford RD, Schatzer R, Wilson BS (2016) A Binaural Cochlear Implant Sound Coding Strategy Inspired by the Contralateral Medial Olivocochlear Reflex. Ear Hear 37:e138.

Marcenaro B, Leiva A, Dragicevic C, López V, Delano PH (2021) The medial olivocochlear reflex strength is modulated during a visual working memory task. J Neurophysiol 125:2309-2321.

Maris E, Oostenveld R (2007) Nonparametric statistical testing of EEG- and MEG-data. J Neurosci Methods 164:177-190. 
Marrufo-Pérez MI, Eustaquio-Martín A, Fumero MJ, Gorospe JM, Polo R, Gutiérrez Revilla A, Lopez-Poveda EA (2019) Adaptation to noise in amplitude modulation detection without the medial olivocochlear reflex. Hear Res 377:133-141.

Mazaheri A, van Schouwenburg MR, Dimitrijevic A, Denys D, Cools R, Jensen O (2014) Region-specific modulations in oscillatory alpha activity serve to facilitate processing in the visual and auditory modalities. Neurolmage 87:356-362.

Mc Laughlin M, Lu T, Dimitrijevic A, Zeng F (2012) Towards a Closed-Loop Cochlear Implant System: Application of Embedded Monitoring of Peripheral and Central Neural Activity. IEEE Trans Neural Syst Rehabil Eng 20:443-454.

Miller CA, Brown CJ, Abbas PJ, Chi S-L (2008) The clinical application of potentials evoked from the peripheral auditory system. Hear Res 242:184-197.

Müller N, Weisz N (2012) Lateralized Auditory Cortical Alpha Band Activity and Interregional Connectivity Pattern Reflect Anticipation of Target Sounds. Cereb Cortex 22:1604-1613.

Oatman LC (1971) Role of visual attention on auditory evoked potentials in unanesthetized cats. Exp Neurol 32:341-356.

O’Brien F, Cousineau D (2014) Representing Error bars in within-subject designs in typical software packages. Quant Methods Psychol 10:56-67.

Oostenveld R, Fries P, Maris E, Schoffelen J-M (2010) FieldTrip: Open Source Software for Advanced Analysis of MEG, EEG, and Invasive Electrophysiological Data Baillet S, ed. Comput Intell Neurosci 2011:156869.

Pedregosa F, Varoquaux G, Gramfort A, Michel V, Thirion B, Grisel O, Blondel M, Prettenhofer P, Weiss R, Dubourg V, Vanderplas J, Passos A, Cournapeau D, Brucher M, Perrot M, Duchesnay É (2011) Scikit-learn: Machine Learning in Python. J Mach Learn Res 12:2825-2830.

Pelli DG (1997) The VideoToolbox software for visual psychophysics: transforming numbers into 
617

618

619

620

621

622

623

624

625

626

627

628

629

630

631

632

633

634

635

636

637

638

639

640

641

movies. Spat Vis 10:437-442.

Prins N, Kingdom FAA (2018) Applying the Model-Comparison Approach to Test Specific Research Hypotheses in Psychophysical Research Using the Palamedes Toolbox. Front Psychol 9.

Ramekers D, Versnel H, Strahl SB, Smeets EM, Klis SFL, Grolman W (2014) Auditory-Nerve Responses to Varied Inter-Phase Gap and Phase Duration of the Electric Pulse Stimulus as Predictors for Neuronal Degeneration. J Assoc Res Otolaryngol 15:187-202.

Riddle J, Scimeca JM, Cellier D, Dhanani S, D'Esposito M (2020) Causal Evidence for a Role of Theta and Alpha Oscillations in the Control of Working Memory. Curr Biol 30:17481754.e4.

Robertson D (1985) Brainstem location of efferent neurones projecting to the guinea pig cochlea. Hear Res 20:79-84.

Sanchez G, Lecaignard F, Otman A, Maby E, Mattout J (2016) Active SAmpling Protocol (ASAP) to Optimize Individual Neurocognitive Hypothesis Testing: A BCl-Inspired Dynamic Experimental Design. Front Hum Neurosci 10.

Somers B, Long CJ, Francart T (2021) EEG-based diagnostics of the auditory system using cochlear implant electrodes as sensors. Sci Rep 11:5383.

Terreros G, Delano PH (2015) Corticofugal modulation of peripheral auditory responses. Front Syst Neurosci 9.

Vallat R (2018) Pingouin: statistics in Python. J Open Source Softw 3:1026.

Varoquaux G, Raamana PR, Engemann DA, Hoyos-Idrobo A, Schwartz Y, Thirion B (2017)

Assessing and tuning brain decoders: Cross-validation, caveats, and guidelines. Neurolmage 145:166-179.

Warr WB, Guinan JJ (1979) Efferent innervation of the organ of corti: two separate systems. Brain Res 173:152-155. 
642

643

644

645

646

647

648

649

650

651

652

653

Weise A, Hartmann T, Schröger E, Weisz N, Ruhnau P (2016) Cross-modal distractors modulate oscillatory alpha power: the neural basis of impaired task performance. Psychophysiology 53:1651-1659.

Weisz N, Hartmann T, Müller N, Obleser J (2011) Alpha Rhythms in Audition: Cognitive and Clinical Perspectives. Front Psychol 2.

Weisz N, Müller N, Jatzev S, Bertrand O (2014) Oscillatory Alpha Modulations in Right Auditory Regions Reflect the Validity of Acoustic Cues in an Auditory Spatial Attention Task. Cereb Cortex 24:2579-2590.

Wetzels R, Wagenmakers E-J (2012) A default Bayesian hypothesis test for correlations and partial correlations. Psychon Bull Rev 19:1057-1064.

Wilson BS, Lawson DT, Müller JM, Tyler RS, Kiefer J (2003) Cochlear Implants: Some Likely Next Steps. Annu Rev Biomed Eng 5:207-249. 
A)

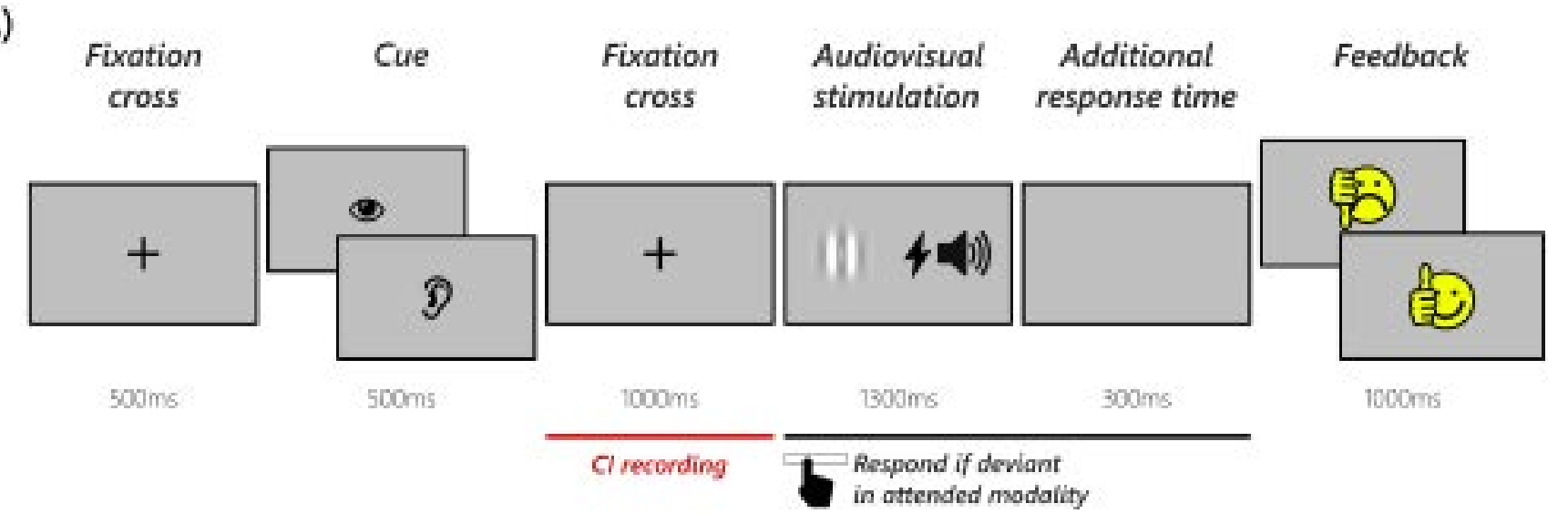

B)

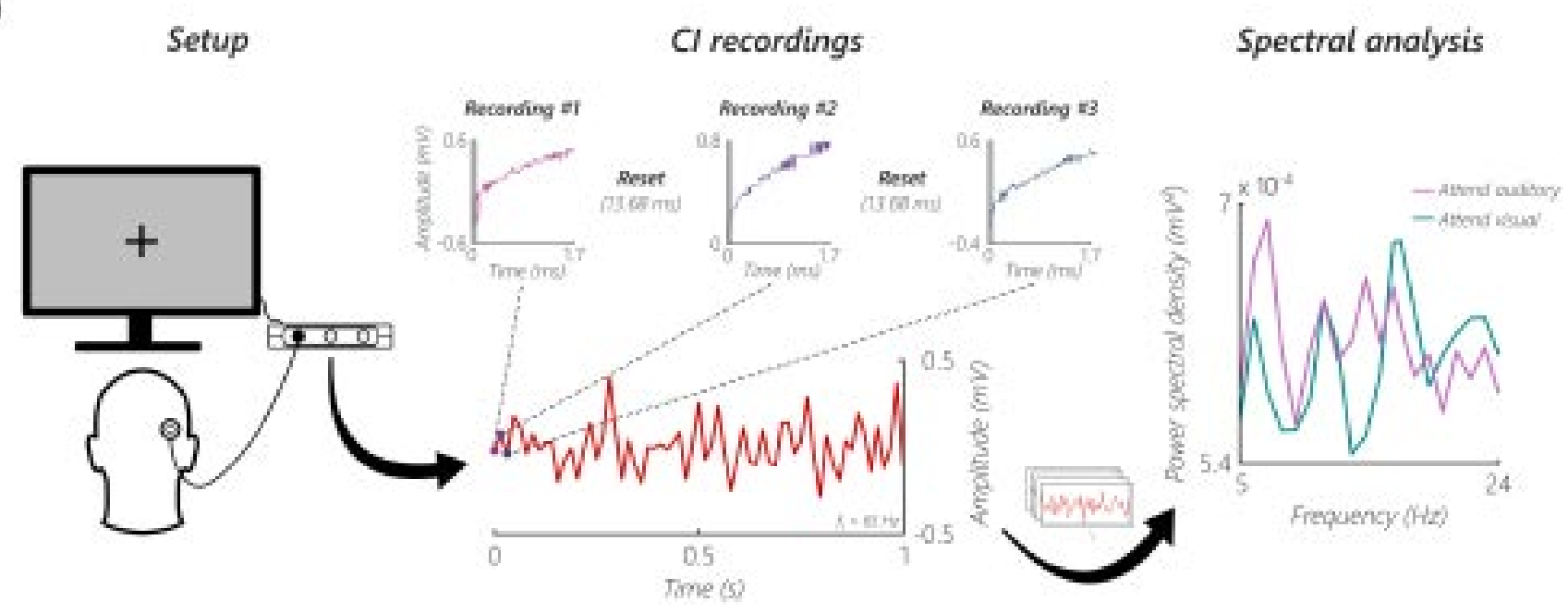


A) Prestimulus power

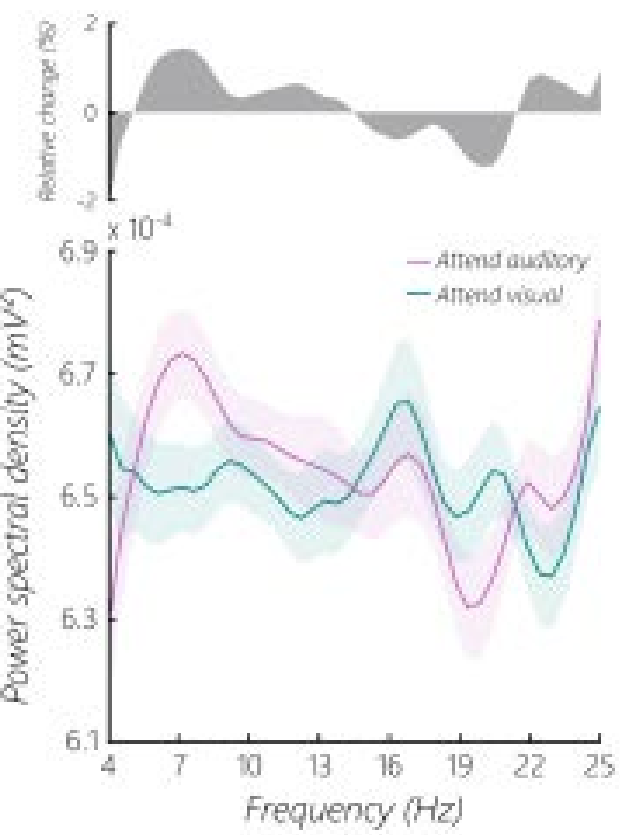

B)

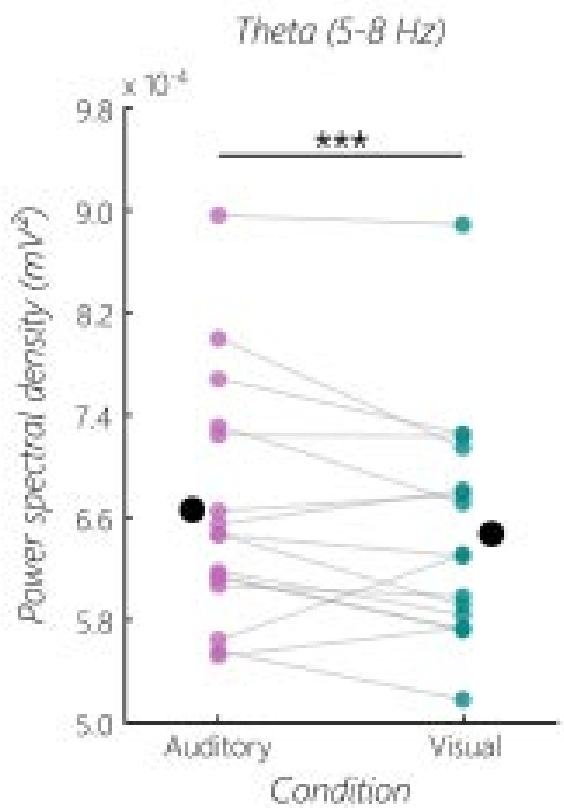

$k N N$-Classification performance

C)

Theta $(5-8 \mathrm{~Hz})$

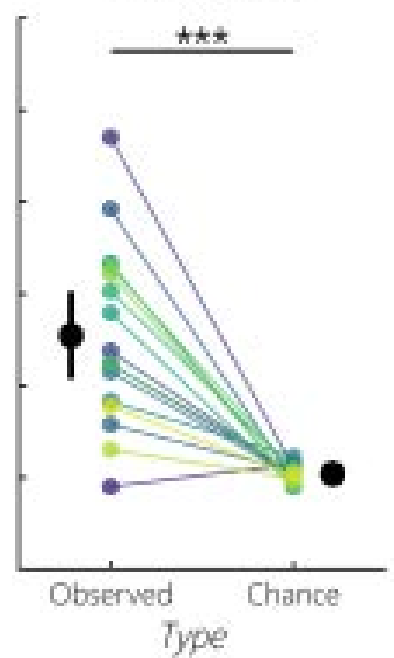

Aipha (9-13 Hz)

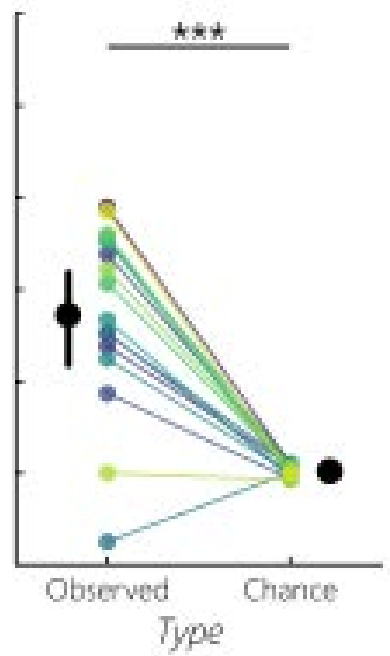

Broadband $(4-25 \mathrm{~Hz})$

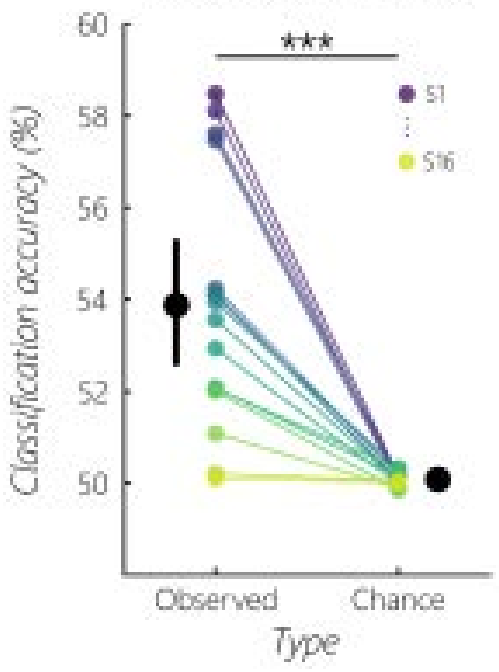

0
0
0
3
7
7
10

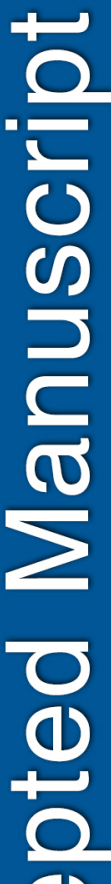

0

0

$<$

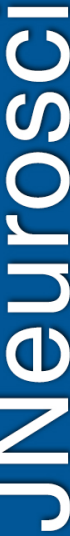

Alpho (9-13 Hz)

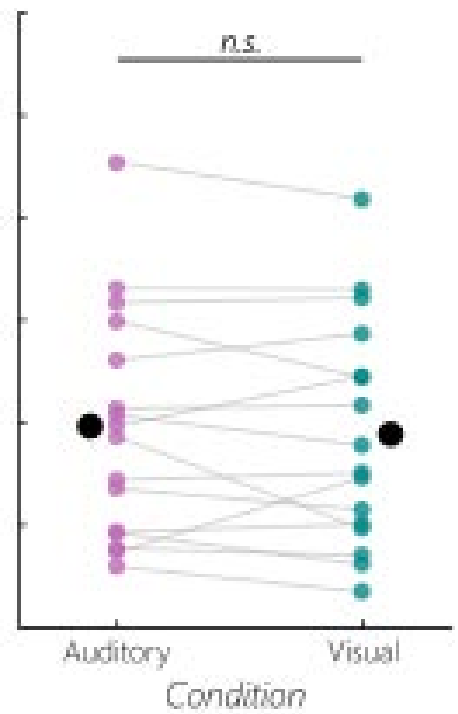

Condition
Auditory vs. Visual

Beto $(74-24 \mathrm{~Hz})$

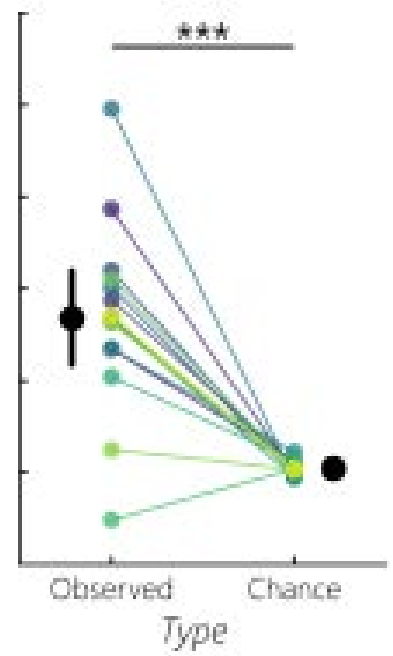

